\title{
复杂曲面通道多轴加工的刀具选择方法
}

\author{
吴宝海 梁满仓张莹罗明 \\ (西北工业大学现代设计与集成制造技术教育部重点实验室＼cjkstart西安７10072)
}

\begin{abstract}
摘要: 针对复杂曲面通道零件多轴加工的刀具优化选择问题, 提出一种基于点可行空间分析的刀具选择方法。分析了复杂曲 面通道多轴加工刀具运动的几何约束, 利用临界约束条件对通道加工的临界刀轴进行求解。通过分析单点可达区域与球头刀 可行摆刀域之间的关系, 提出一种无碰撞干涉条件下的最大刀具直径选择方法; 在此基础上, 通过对可行摆刀域内刀轴矢量 的优化, 获得了刀具直径最大条件下的最短刀具长度。以整体叶轮的五轴加工为例, 对该方法进行了分析与验证, 给出了叶 片曲面上最大刀具直径与最短刀具长度的变化情况, 并对不同刀具直径的加工效果进行了对比分析。结果表明, 本文方法能 获得复杂曲面通道类零件多轴加工的最大刀具直径、最小刀具长度, 可显著提高该类零件的加工效率及加工稳定性。
\end{abstract}

关键词: 复杂曲面通道; 刀具选择; 球头刀; 碰撞干涉

中图分类号: TP391

\section{Tool Selection of Multi-axis Machining for Channel Parts with Sculptured Surface}

\author{
WU Baohai LIANG Mancang ZHANG Ying LUO Ming
}

(Key Laboratory of Contemporary Design and Integrated Manufacturing Technology of Ministry of Education, Northwestern Polytechnical University, Xi'an 710072)

\begin{abstract}
This paper is devoted to selecting an optimal ball-end tool for machining channel parts with sculptured surface on the multi-axis machining. A method is proposed that based on analyzing the feasible tool orientation of a given single point. Critical tool directions are obtained by establishing and analyzing the mathematic model of cutting tool motion. After researching the differences between visibility direction on the single point and feasible tool orientation of a given ball-end, maximum tool size can be calculated without collision between tool and workpiece. Furthermore, minimum tool length is obtained according to optimize the feasible tool orientation after selecting maximum tool size. Finally, an impeller is machined in a five-axis machining to verify the validity and correctness of the presented approach. Meanwhile, the changes of maximum tool size and minimum tool length on the whole machined surface are given. The machining results used different tools are compared and analyzed. The results suggest that the maximum tool size and minimum tool length can be achieved for machining channel parts with sculptured surface by implementing the developed method, and the machining efficiency and machining stability can be significantly improved.
\end{abstract}

Key words: channel parts with sculptured surface; cutter selection; ball-end cutter; collision and interference

\section{0 前言}

复杂曲面通道零件，如整体叶盘、叶轮等广泛 应用于航空航天、动力能源等领域, 其加工质量直 接决定了该类装备的运行性能。这类零件结构复杂、 通道扭曲狭长, 叶片超薄且弯扭严重, 且材料多为 钛合金、高温合金等难加工材料, 因此加工效率低、 工艺稳定性差一直是制约该类零件生产的瓶颈问

* 国家重大科技专项资助项目(2015ZX04001202)。20170211 收到初稿, 20170519 收到修改稿
题。多轴数控加工是这类零件制造的主要工艺手段, 刀具直径和长度是制约其加工效率和加工过程稳定 性的关键要素 ${ }^{[1-2]}$ 。如何根据零件的几何特性合理地 选择刀具是提高加工效率和加工过程稳定性的关键 环节。

通常情况下, 大的刀具直径有助于提高加工效 率和加工稳定性, 同时也增加了干涉发生的可能性; 而小的刀具直径虽然有助于干涉的避免, 但加工过 程极易发生断刀等风险, 且加工效率低。为此, LI 等 ${ }^{[3]}$ 提出一种基于曲面分解的刀具选择方法, 通过 比较待加工曲面和刀具之间的微分几何特性避免局 
部干涉, 该方法具有较高的计算效率。DING 等 ${ }^{[4]}$ 通过计算待加工曲面最大主曲率来确定无曲率干涉 的最大刀具直径。然而, 文献 [3-4]没有考虑到碰撞 干涉对刀具尺寸的影响。为此, JENSEN 等 ${ }^{[5]}$ 综合 考虑局部干涉和全局干涉的避免, 针对环形刀提 出一种基于曲率匹配的刀具直径优化选择方法。 LI 等 ${ }^{[6]}$ 提出一种针对给定的环形刀是否能完成精加 工整个曲面的判别方法, 首先将加工曲面离散为特 征点集, 考虑到机床运动轴极限、曲率干涉、后角 干涉以及全局干涉的避免, 确定了单点处刀轴可行 空间, 若待加工曲面上所有特征点处的刀轴可行空 间存在交集, 则给定刀具能胜任整个曲面的加工, 但该方法计算量较大, 且没有综合考虑刀具长度。 GLAESER 等 ${ }^{[7]}$ 研究了三轴加工中避免局部干涉和 碰撞干涉的刀具选择方法, 包括刀具的设计原则等, 但不适用于五轴联动加工。YANG 等 ${ }^{[8]}$ 通过一套路 径生成方法模拟加工过程, 进行三轴加工的干涉检 查和刀具优选, 但所采用的优选方法实际是穷举比 较的过程。

就刀具长度而言, 刀具长度对加工稳定性有着 至关重要的影响。较短的刀具长度不易产生振动、 刀具刚性好。但是, 选用较短的刀具长度导致机床 主轴刀具加持部分与工件间容易发生碰撞干涉。为 此, JENSEN 等 ${ }^{[5]}$ 通过检验碰撞干涉发生的位置, 确定了刀具最小有效长度。MORIMOTO 等 ${ }^{[9]}$ 通过规 划刀轴矢量来优化刀具长度, 但干涉检测方法过于 保守, 且不能用于多轴联动数控加工中。综上所述, 现有的刀具直径选择方法基本上都是通过研究目标 曲面与刀具形状之间的局部匹配关系来实现最大刀 具尺寸的选择, 且很少综合考虑刀具长度, 不适于 加工碰撞干涉大量存在的复杂曲面通道类零件。另 一方面, 五轴数控加工中, 机床自由度的增加使得 所选刀具半径大于待加工曲面的最小曲率半径成为 可能 ${ }^{[10]}$ 。因此, 通过计算待加工曲面曲率实现最大 刀具尺寸选择的方法 ${ }^{[3-6]}$ 具有一定的局限性。实际 上, 刀具选择主要考虑到局部干涉和碰撞干涉的避 免, 局部干涉可以通过研究目标曲面与刀具形状之 间的局部匹配关系予以避免 ${ }^{[11]}$ 。而由于碰撞干涉发 生位置的不确定性以及计算的复杂性导致其不易进 行判断。WANG 等 ${ }^{[12]}$ 利用 $\mathrm{C}$ 空间法求解了给定切触 点处无碰撞干涉刀轴摆动范围。尹周平等 ${ }^{[13]}$ 发展了 可视雉方法, 可用于计算可行刀轴空间。LI 等 ${ }^{[14]}$ 通过设置参考平面提出一种二维可行摆刀域分析方 法, 有效提高了计算效率。

基于以上分析, 为提高复杂曲面通道零件的加 工效率和加工过程稳定性, 本文针对球头刀, 综合
考虑刀具直径和长度, 通过计算刀轴矢量可行空间, 建立刀具几何参数优化模型, 提出一种能获得最大 刀具直径、最小刀具长度且碰撞干涉避免的刀具优 化选择方法, 同时, 优化刀具的可行摆刀范围也随 之确定, 为之后的刀具路径规划和刀轴矢量优化提 供了有效可靠的基础信息。

\section{1 不带直径刀具可行摆刀范围建立}

\section{1 多轴加工刀具运动几何约束}

多轴数控加工中, 如图 1 所示, $S_{0}$ 为待加工曲 面, $n_{c}$ 为切触点 $C$ 处的曲面法矢量方向, $\boldsymbol{f}_{c}$ 为 $C$ 点 的走刀方向, 由几何关系知 $\boldsymbol{f}_{\boldsymbol{c}} \perp \boldsymbol{n}_{\boldsymbol{c}}$ 。为避免局部干 涉, 刀轴摆动范围应限制在点 $\boldsymbol{C}$ 的切平面 $P_{c}$ 之上。 为形成拖刀加工, 刀轴摆动范围被进一步限定在与 走刀方向垂直的临界平面 $P_{0}$ 与切平面 $P_{c}$ 之间。本文 中给定切触点处可行刀轴摆动范围将在该区域内进 行研究。

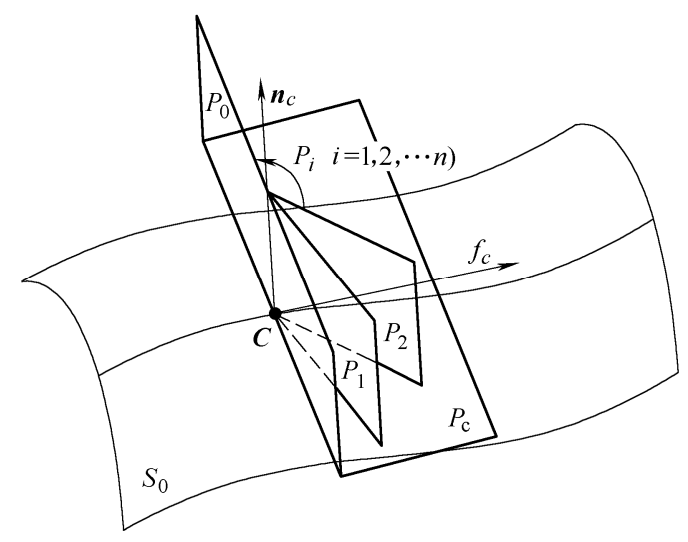

图 1 多轴加工刀具运动几何约束

\section{2 临界刀轴约束求解}

首先, 不考虑刀具几何形状, 将其抽象为一条 射线, 则可以通过研究切触点 $\boldsymbol{C}$ 的可视性代替刀具 可达性分析 ${ }^{[13]}$ 。将临界平面 $P_{0}$ 与切平面 $P_{c}$ 之间的刀 轴允许摆动范围离散为若干个可行摆刀平面 $P_{i}(i=1,2, \cdots, n)$ 。临界刀轴约束是指刀杆受潜在干涉曲 面上临界元素(点、曲线、曲面等)的约束, 只能在 一定范围内运动而不能超越这一临界元素的一种约 束方式 ${ }^{[15]}$ 。在临界摆刀平面 $P_{i}$ 中, 临界元素为检查 曲面与当前摆刀平面 $P_{i}$ 相交曲线上一点, 称为临界 点。临界刀轴矢量即为由切触点指向临界点的单位 向量。则将刀轴可行摆刀范围离散化之后, 对临界 刀轴约束的求解将转化为干涉曲面与当前摆刀平面 相交曲线上临界点的求解。

加工复杂曲面通道类零件时, 待加工曲面与其 相邻曲面均有可能对刀轴的摆动造成约束。计算待 
加工曲面上刀轴的临界约束, 即是求待加工曲面与 当前摆刀平面相交曲线上临界点的过程。如图 2 所 示, 在可行摆刀平面 $P_{i}$ 上, 由几何知识可得, 切触 点 $\boldsymbol{C}$ 与临界点 $\boldsymbol{M}_{0}$ 连线构成的单位向量 $\boldsymbol{T}_{0}$ 与曲面法 矢 $\boldsymbol{n}_{\mathrm{c}}$ 之间的夹角 $\theta$ 最小, 则求解临界点的模型可表 示为 ${ }^{[16]}$

$$
\max \left\{\frac{\left(\boldsymbol{c}_{\boldsymbol{i}}(t)-\boldsymbol{I}_{\boldsymbol{c}}\right) \cdot \boldsymbol{n}_{\boldsymbol{c}}}{\left(\boldsymbol{c}_{\boldsymbol{i}}(t)-\boldsymbol{I}_{\boldsymbol{c}}\right) \cdot \boldsymbol{f}_{\boldsymbol{c}}}\right\}
$$

$\boldsymbol{c}_{\boldsymbol{i}}(t)$ 为当前摆刀平面与待加工曲面的相交曲 线方程, 以弧长 $t$ 为参数, $I_{c}$ 为当前切触点 $C$ 的位 置矢量。本文利用临界刀轴表示刀具无碰撞干涉区 域, 碰撞干涉是一个全局问题, 故研究切触点邻域 对本问题没有意义, 令 $\boldsymbol{c}_{i}(t) \notin\left[\boldsymbol{I}_{c}-\delta, \boldsymbol{I}_{c}+\delta\right]$ 可避免上 式中分母为零的情况。

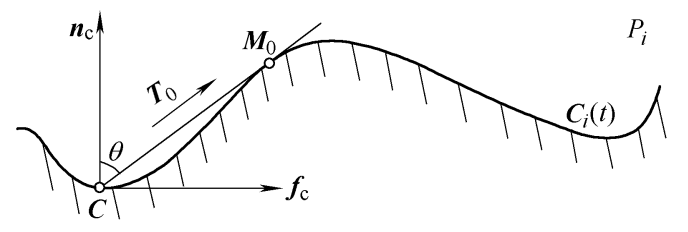

图 2 待加工曲面临界点计算

反之, 对于潜在干涉曲面上的刀轴临界约束, 其临界刀轴单位向量 $\boldsymbol{T}_{1}$ 与上述所求待加工曲面上 的临界刀轴矢量 $\boldsymbol{T}_{0}$ 之间的夹角 $\theta$ 最小, 如图 3 所示, 则临界点的搜索模型变为 ${ }^{[16]}$

$$
\max \left\{\frac{\left(\boldsymbol{c}_{i}^{\prime}(t)-\boldsymbol{I}_{c}\right) \cdot \boldsymbol{T}_{0}}{\left|\boldsymbol{c}_{i}^{\prime}(t)-\boldsymbol{I}_{c}\right|\left|\boldsymbol{T}_{0}\right|}\right\}
$$

$\boldsymbol{c}_{i}^{\prime}(t)$ 为当前摆刀平面与潜在干涉曲面之间的 相交曲线方程, 以弧长 $t$ 为参数。

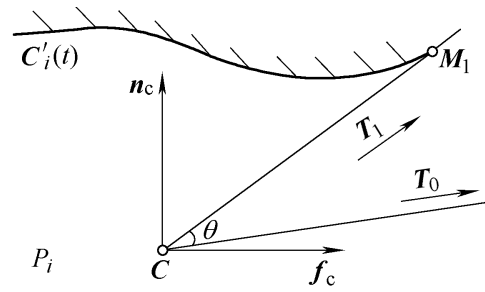

图 3 潜在干涉曲面临界点计算

\section{2 球头刀可行摆刀域计算}

\section{1 球头刀可行摆刀域与可达区域分析}

前面的讨论中, 刀具被抽象为一条无限长的射 线, 给定点处的可行摆刀域即为该点的可达区域, 如图 4a 所示。然而, 由于刀具尺寸的影响, 球头刀 的可行摆刀域实际上是可达区域的子集, 如图 $4 \mathrm{~b}$ 所示。进一步, 当刀具与待加工曲面仅在切触点处
切触时, 刀具半径应该小于或等于其与检查曲面之 间不发生干涉的最近距离, 从而最大刀具半径可以 用刀具轴线到检查曲面之间的最短距离近似代替。 应该指出，对于半径为 $R$ 的球头刀而言，可以将待 加工曲面与潜在干涉曲面分别沿外法矢方向偏置距 离 $R$ 从而消除刀具直径对计算结果的影响。

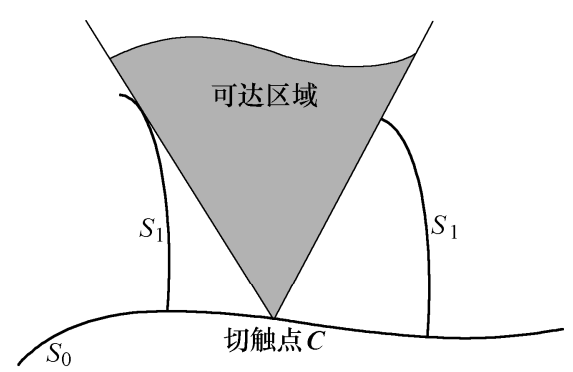

(a) 可达区域

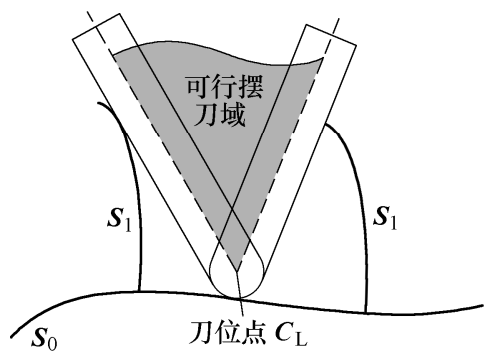

(b) 球头刀可行摆刀域

图 4 可达区域与可行摆刀域之间的关系

目前, 在计算机复杂形状几何造型中, NURBS 曲面由于具有较统一的数学形式, 以及具有强大的 局部调控能力而被广泛使用。本文中采用 NURBS 曲面描述待加工曲面 $S_{0}$ 和潜在干涉曲面 $S_{1}$, 其方程 分别表示为 $S_{0}(u, v)$ 和 $S_{1}(u, v)$ 。其各自的偏置曲面即 是对源曲面进行等距变换, 即将源曲面上每一点沿 其各自的外法矢 $\boldsymbol{n}$ 方向偏置距离 $R$, 从而可得到曲 面 $S_{0}(u, v)$ 和 $S_{1}(u, v)$ 的等距面方程

$$
\begin{aligned}
& S_{0}^{\prime}(u, v)=S_{0}(u, v)+R \boldsymbol{n} \\
& S_{1}^{\prime}(u, v)=S_{1}(u, v)+R \boldsymbol{n}
\end{aligned}
$$

进一步, 根据前述分析, 即可求出半径为 $R$ 的 球头刀在给定切触点处的临界刀轴矢量 $\boldsymbol{T}$ 。

\section{2 球头刀可行摆刀域求解}

以工件坐标系原点 $O_{\mathrm{w}}$ 为球心作一单位球面, 称为待加工曲面 $S_{0}$ 的高斯球面 ${ }^{[17]}$ 。将切触点 $\boldsymbol{C}$ 处的 所有临界刀轴单位矢量 $\boldsymbol{T}$ 平移至球心点 $O_{\mathrm{w}}$, 则每 一个临界刀轴矢量 $\boldsymbol{T}$ 将被映射到高斯球面上一点 $\boldsymbol{P}$, 如图 5 所示, 所有点 $\boldsymbol{P}$ 构成的集合组成了切触 点 $C$ 处的可行摆刀域 $\Omega$

$$
\Omega=\{\boldsymbol{P} \mid \boldsymbol{P}=\boldsymbol{T}\}
$$




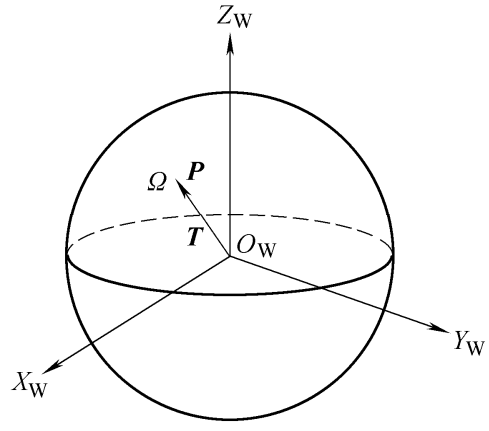

图 5 临界刀轴矢量高斯映射

\section{3 刀具优化选择}

多轴数控加工中, 给定待加工曲面上一点, 如 果指定刀具能无干涉地到达该点, 则认为刀具能胜 任该点的加工。如果刀具能无干涉地到达待加工曲 面上任意一点, 则认为该刀具能用于加工整个曲面。 本节致力于寻找能实现整个待加工曲面无碰撞干涉 加工的最优球头刀具, 为简化计算, 首先将待加工 曲面离散为若干特征点, 针对每个特征点进行可行 空间分析, 按照刀具直径尽可能大, 刀具长度尽可 能短的优化目标，找出能实现各个特征点无碰撞干 涉存在的最优刀具参数, 最后将针对单点的可行空 间分析推广至整个待加工曲面, 在所有特征点处的 最大刀具尺寸中选择最小值, 所有最短刀具长度中 选择最大值作为所选刀具的最终优化参数。

\section{1 单点处刀具参数优化算法}

降低刀具的长径比, 有助于提高加工效率和加 工质量。根据前面分析, 在不考虑刀具长度的情况 下, 判断半径为 $R$ 的球头刀是否对于给定的切触点 $C$ 是可达的，首先将点 $C$ 所在的待加工曲面以及与 其相邻的潜在干涉曲面沿外法矢方向偏置距离 $R$, 其次计算在一系列偏置曲面的约束条件下，刀位点 处的临界刀轴, 将所有临界刀轴映射到高斯球面上, 若投影区域非空, 则该球头刀在切触点 $\boldsymbol{C}$ 处具有可 达方向, 即存在可行的刀轴矢量, 使得半径为 $R$ 的 球头刀可以无碰撞干涉地到达点 $\boldsymbol{C}$ 。

在此基础上, 对刀具的有效长度进行计算。复 杂曲面通道类零件多轴数控加工中, 刀具长度一般 由刀具方向决定, 本文中沿着刀具可行方向计算安 全最短刀具长度。定义球头刀刀具长度 $L=R+D$, 其中, $R$ 为刀具半径, $D$ 为刀杆长度。以图 $6 \mathrm{a}$ 中 的通道零件模型为例, 其他类型的复杂曲面通道可 按照本文方法进行同样的分析。工件坐标系 $O_{\mathrm{w}} X_{\mathrm{w}} Y_{\mathrm{w}} Z_{\mathrm{w}}$ 中, 刀具可以从上方、下方以及外侧三 个区域接近零件, 则定义上约束平面 $Z_{\mathrm{w}}=Z_{1}$, 下约
束平面 $Z_{\mathrm{w}}=Z_{2}$, 圆柱面 $X_{\mathrm{w}}^{2}+Y_{\mathrm{w}}^{2}=r^{2}$, 以及回转曲 面 $\operatorname{rot}^{\prime}(t, \theta)$ 。其中曲面 $\operatorname{rot}^{\prime}(t, \theta)$ 为叶尖曲线 $\operatorname{cur}(t)$ 绕 $Z_{\mathrm{W}}$ 轴旋转一周后所得到的回转面 $\operatorname{rot}^{\prime}(t, \theta)$ 的偏 置面。可将上、下约束平面、回转曲面 $\operatorname{rot}^{\prime}(t, \theta)$ 和 圆柱面构成的区域作为计算刀具长度的约束面，则 刀杆长度即可以表示为刀位点到刀轴线与约束面交 点之间的距离。如图 $6 \mathrm{~b}$ 所示, 刀位点 $\boldsymbol{O}_{L 1}$ 和刀轴矢 量 $\boldsymbol{T}_{1}$ 所确定的刀具姿态受到回转曲面 $\operatorname{rot}^{\prime}(t, \theta)$ 的 约束, 可定义如下刀长求解模型

$$
L=R+D_{1}
$$

$D_{1}$ 为刀位点 $\boldsymbol{O}_{L 1}$ 到刀具轴线与回转曲面 $\operatorname{rot}^{\prime}(t, \theta)$ 交点 $B_{1}$ 之间的距离。

当刀具姿态受上、下约束面限制时，如图 $6 \mathrm{~b}$ 中 刀位点 $\boldsymbol{O}_{L 2}$ 和刀轴矢量 $\boldsymbol{T}_{2}$ 所确定的刀具姿态, $Z_{\mathrm{L} 2}$ 为 刀位点 $\boldsymbol{O}_{L 2}$ 在 $Z_{\mathrm{w}}$ 轴上的坐标, 刀长求解模型变为

$$
\begin{gathered}
L=R+D_{2} \\
D_{2}=d / \cos \alpha \\
d=Z_{1}-Z_{L 2} \quad \cos \alpha>0 \\
d=Z_{2}-Z_{L 2} \quad \cos \alpha \leqslant 0
\end{gathered}
$$

$\cos \alpha>0$, 即刀轴矢量 $\boldsymbol{T}_{2}$ 与 $Z_{\mathrm{w}}$ 轴正方向一致, 故刀具长度受上约束面 $Z_{\mathrm{W}}=Z_{1}$ 的限制;

$\cos \alpha \leqslant 0$, 即刀轴矢量 $\boldsymbol{T}_{2}$ 与 $Z_{\mathrm{w}}$ 轴负方向一致, 故刀具长度受下约束面 $Z_{\mathrm{w}}=Z_{2}$ 的限制。

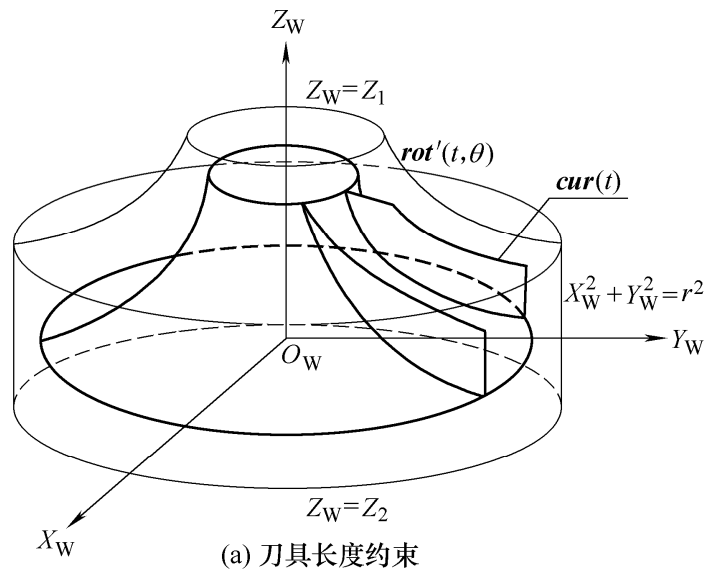

(a) 刀具长度约束

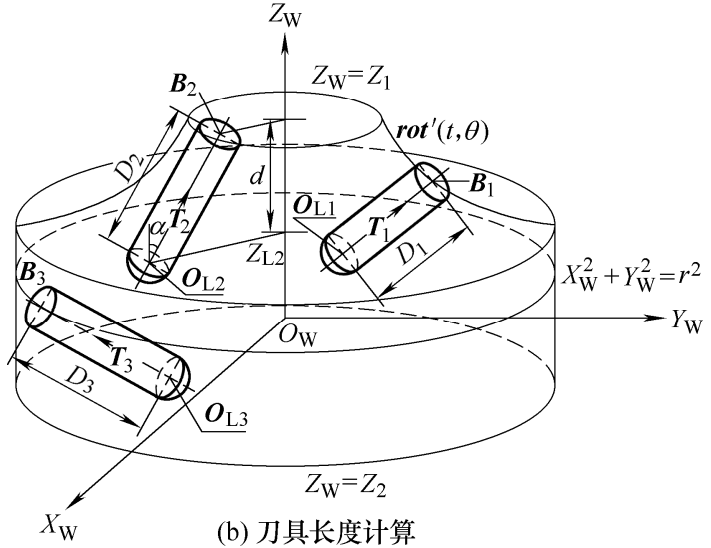

(b) 刀具长度计算

图 6 刀具有效长度计算模型 
应该指出, 上述计算刀具最大直径和有效最短 刀具长度时, 两者对应的刀轴方向可能不同, 为平 衡二者之间的关系, 同时保证优化后的刀具直径和 刀具长度尽量接近于最大刀具直径和最小刀具长 度, 考虑数据对 $\left(R_{i}, L_{i}\right)$ 与理论最优值 $\left(R_{\text {max }}, L_{\text {min }}\right)$ 之 间的偏离程度最小, 参照方差定义取优化目标函数 $f\left(R_{i}, L_{i}\right)=\min _{\text {all } T}\left(\left[\alpha\left(2 R_{i}-2 R_{\max }\right)^{2}+(1-\alpha)\left(L_{i}-L_{\min }\right)^{2}\right]\right)$

式中 $R_{\text {max }}$ 一给定切触点处所有允许选用的刀具 半径 $R_{i}$ 中的最大值;

$L_{\text {min }}$ 一给定切触点处所有刀轴可行方向对 应的刀具长度 $L_{i}$ 中的最小值;

$\alpha$ 一平衡系数(工艺人员按实际经验给出)。

目标函数 $f\left(R_{i}, L_{i}\right)$ 取值最小时, 优化值 $R_{i}$ 和 $L_{i}$ 最接近于最优值 $R_{\text {max }}$ 和 $L_{\text {min }}$ 。

给定点处刀具直径和刀具长度参数优化算法流 程如下。

(1) 输入待加工曲面 $S_{0}$, 潜在干涉曲面 $S_{1}$ (可能 有多个), 切触点 $C$, 初始化刀具半径 $R_{0}=R_{\text {min }}$, 增 量 $\Delta R$, 平衡系数 $\alpha, n=0, i=0$ 。

(2) 将待加工曲面 $S_{0}$, 潜在干涉曲面 $S_{1}$ 沿其各 自的外法线方向偏置距离 $R_{i}$, 同时计算切触点 $\boldsymbol{C}$ 所 对应的刀位点 $\boldsymbol{O}_{L}$ 。

(3) 计算刀位点 $\boldsymbol{O}_{L}$ 在各个偏置曲面约束下的临 界刀轴单位矢量 $\boldsymbol{T}$ 。

(4) 将各个临界刀轴单位矢量 $\boldsymbol{T}$ 映射到高斯球 面上, 得到可行摆刀域 $\Omega$ 。

(5) 计算可行摆刀域 $\Omega$ 中各个单位刀轴矢量对 应的刀具长度 $L_{i t}(t=1,2, \cdots, m)$, 取最小值作为安全最 短刀具长度 $L_{i}=\min _{1 \leqslant t \leqslant m} L_{i t}$ 。

(6) 判断 $\Omega$ 是否为空, 若 $\Omega$ 非空, $R_{i+1}=R_{i}+\Delta R$, $n=n+1$, 返回步骤(1); 若 $\Omega$ 为空, 令 $R_{\text {max }}=R_{i}$ 。

(7) 计算 $f\left(R_{i}, L_{i}\right)$ 并输出优化后的刀具半径 $R_{i}$ 和刀具长度 $L_{i}$, 其中 $L_{\min }=\min _{1 \leqslant i \leqslant n} L_{i}$, 算法结束。

实际上, 在第(5)步中, 没必要对每次计算出的 可行摆刀域 $\Omega$ 中的全部刀轴矢量进行对应的刀具 长度计算, 因为随着偏置距离的增大, 可行摆刀域 必然将不断减小, 偏置距离为 $R_{i+1}$ 时的可行摆刀域 是偏置距离为 $R_{i}$ 时的子集(如图 4 所示)。

\section{2 曲面刀具参数优化算法}

将以上针对单点的刀具参数优化算法推广至整 个待加工曲面, 首先将被加工曲面离散为若干特征 点 $\boldsymbol{D}_{j k}(j=1,2, \cdots, p ; k=1,2, \cdots, q)$ 。若所有特征点 $\boldsymbol{D}_{j k}$ 都是 可加工的, 则由特征点集构成的所有参数曲线也必 是可加工的, 则由参数曲线构成的曲面即是可加工
的。基于这一原则, 可按照图 7 的方式将曲面进行 离散化处理, 得到特征点集 $\boldsymbol{D}_{j k}(j=1,2, \cdots, p ; k=$ $1,2, \cdots, q)$ 。然后, 针对每个特征点进行刀具参数优 化, 计算各个特征点所允许选用的最大刀具尺寸和 最短刀具有效长度, 取所有特征点处最大刀具尺寸 中的最小值, 所有特征点处最短刀长中的最大值作 为最终优化结果。算法流程如下。

(1) 在待加工曲面上选取若干特征点 $\boldsymbol{D}_{j k}(j=1$, $2, \cdots, p ; k=1,2, \cdots, q)$ 。

(2) 初始化球头刀半径值 $R_{j k}$ 。

(3) 在每个特征点 $\boldsymbol{D}_{j k}$ 处进行刀具参数优化, 得 到每个特征点处刀具优化参数值 $R_{j k}, L_{j k}$ 。

(4) 在所有 $R_{j k}$ 中选取最小值 $R=\min _{\substack{1 \leqslant j \leqslant \\ 1 \leqslant k \leqslant q}} R_{j k}$, 在所 有 $L_{j k}$ 中选取最大值 $L=\max _{\substack{1 \leqslant i \leqslant p \\ 1 \leqslant k \leqslant q}} L_{j k}, R, L$ 即为可以实 现无碰撞干涉加工整个曲面的最优刀具尺寸参数 值，算法结束。

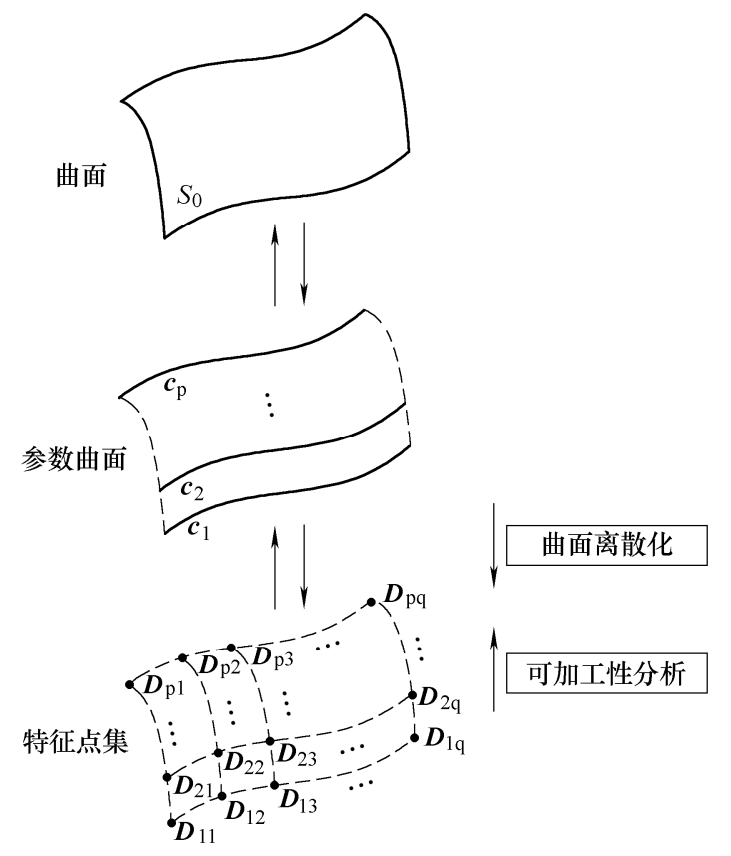

图 7 曲面离散与可加工性分析示意图

下面对本文算法的时间复杂度进行评估。由于 本文中刀具长度是在刀具直径确定的条件下, 通过 对可行摆刀域内刀轴矢量进行优化而得到的, 因此, 以可行摆刀域内各个刀轴矢量对应的刀具长度计算 为基本操作, 以该基本操作在算法中重复执行的次 数为算法运行时间的衡量准则, 当问题规模为 $m$ 时 (即曲面被离散为 $m$ 个特征点), 由于给定点处刀具 半径 $R_{i+1}$ 所确定的可行摆刀域是刀具半径为 $R_{i}$ 时 的子集(图 4), 因此, 对于确定的刀具半径, 上述 基本操不必重复执行，本文算法时间复杂度可近 似表示为 


$$
\sum_{j=1}^{m} \sum_{i=1}^{n} 1=\sum_{j=1}^{m} n=m n
$$

式中, $n$ 为曲面偏置次数。

则本文算法的时间复杂度为线性级 $O(m)$ 。

应该指出, 对于一些复杂曲面通道, 如整体叶 轮通道而言, 由于通道进口宽度明显小于出口宽度, 为减少计算量, 只需要在通道进气边宽度较窄的部 分提取适当特征点进行计算即可。

\section{4 仿真分析与验证}

为了验证所研究方法的正确性及有效性, 以整 体叶轮通道为例, 利用 UG 软件二次开发并结合 Matlab 软件编程对本文算法进行实现, 叶轮通道模 型如图 8 所示。首先, 当不考虑刀具直径时, 根据 所描述的刀具运动几何约束求解单个切触点处的临 界刀轴矢量 $\boldsymbol{T}$, 如图 9 所示, 图中紫色刀轴为避免 待加工曲面本身及相邻曲面干涉所生成的临界刀 轴, 绿色刀轴为避免顶刀加工和轮款干涉所生成的 临界刀轴, 在图 9a 中, 切触点靠近叶根且位于叶身 中部, 受加工曲面、轮㪍面和相邻叶片面影响均较 大，所以临界刀轴在三者上的分布范围均较大; 图 $9 \mathrm{~b}$ 中, 切触点位于通道排气边一侧, 相对于图 $9 \mathrm{a}$ 而言, 开敞性有所改善, 刀轴摆动主要受限于待加 工曲面和轮毂曲面约束, 在相邻叶片曲面上的分布 范围较小。

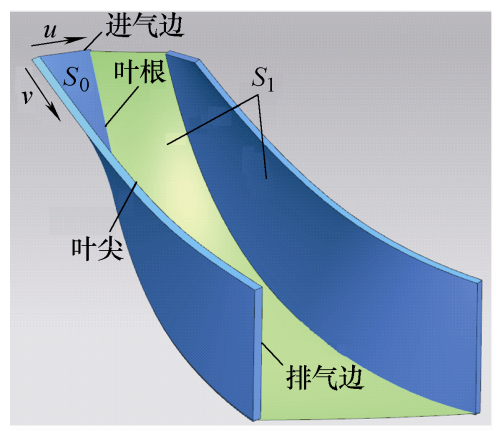

图 8 整体叶轮通道模型

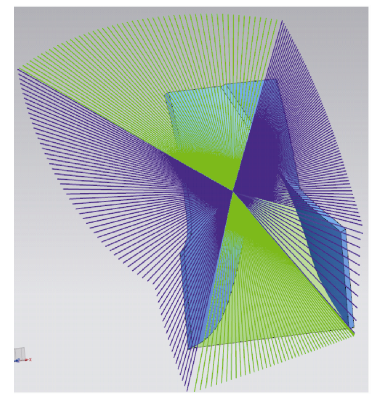

(a) $u=0.75, v=0.34$

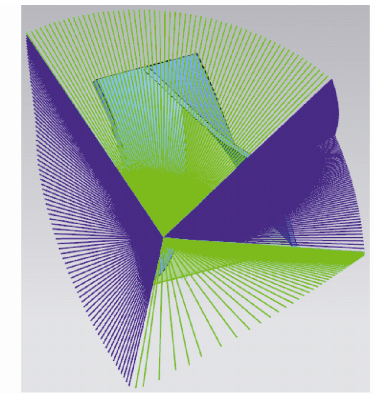

(b) $u=0.75, v=0.78$
图 9 单点处临界刀轴矢量
初始化刀具半径 $R_{0}=0$, 增量 $\Delta R=0.05 \mathrm{~mm}$, 平 衡系数 $\alpha=0.5$, 刀具长度约束面分别取距叶轮上端 面、下端面以及叶尖曲线所构成的回转面各偏置 10 $\mathrm{mm}$ 的三个面, 按照本文提出的刀具参数优化算法 进行分析, 得到加工该叶轮通道曲面的球头刀最大 可用刀具半径为 $7 \mathrm{~mm}$, 最短有效刀具长度为 76 $\mathrm{mm}$, 其中最大刀具半径在 $u=0.75, v=0.17$ 处取 得, 最短刀长在加工曲面上 $u=0.75, v=0.58$ 处取 得。在点 $u=0.45, v=0.805$ 处, 球头刀半径变化与 可行摆刀域之间的关系如图 10 所示, 可见随着刀具 半径增大，碰撞干涉发生的概率增加，从而可行摆 刀域将减小。

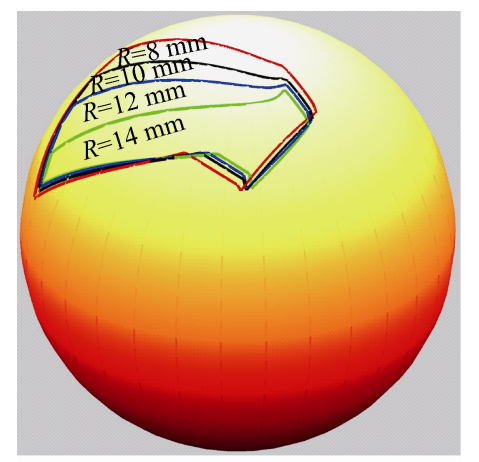

图 10 球头刀半径与可行摆刀域之间的关系

图 11、12 分别给出了整张待加工曲面上刀具最 大直径与最短刀具长度变化情况, 分析图 8 中模型 可知, 叶尖部分、以及通道的上下两侧开敞性比较 好, 可选取较大刀具直径, 且可行刀轴方向较多, 可选用较短刀具长度, 而叶根部分受约束曲面影响 较大, 导致最大可用刀具直径减小, 为避免碰撞干 涉, 刀具悬伸量较大，从而刀具长度较大。例如曲 面参数 $u=0.3$ 时，随着参数 $v$ 增大，即从进气边到 排气边的过程, 最大刀具直径先减小后增大, 最短 刀具长度先增大后减小; 曲面参数 $v=0.6$ 时, 随着 参数 $u$ 增大, 即从叶尖到叶根的过程, 通道深度加 大, 受相邻曲面的影响增大, 碰撞干涉发生的概率 增大, 从而最大刀具直径逐渐减小, 最短刀具长度 则逐渐增大。实际情况与分析结果相符, 证明了本 文方法的正确性。

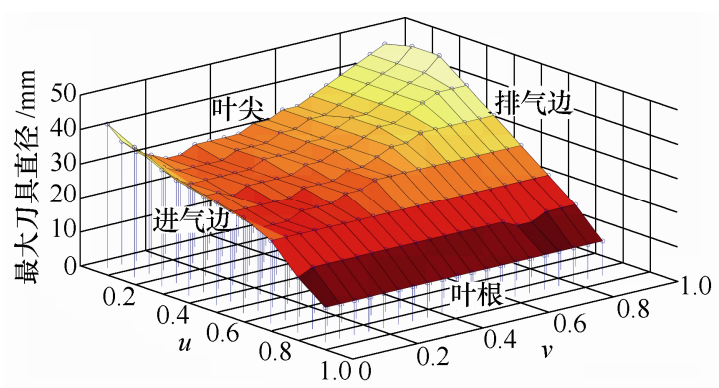

图 11 待加工曲面最大刀具直径变化 


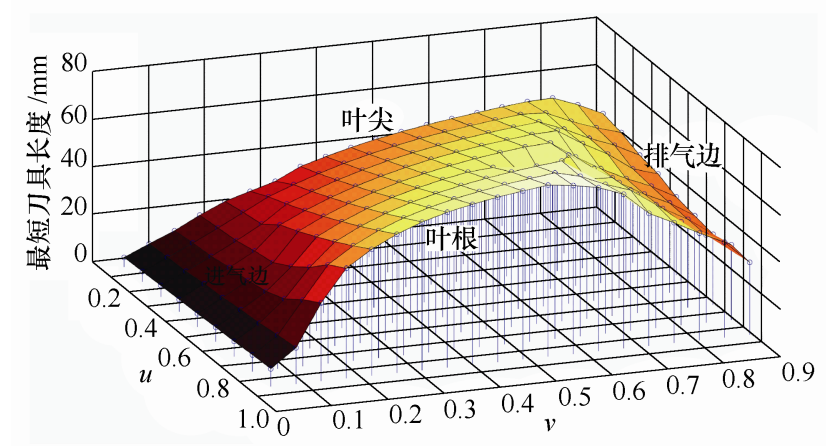

图 12 待加工曲面最短刀具长度变化

为了验证不同刀具直径对加工结果的影响, 本 文采用未经优化的刀具直径 $10 \mathrm{~mm}$ 和优化后的刀具 直径 $14 \mathrm{~mm}$ 对加工路径进行了规划。利用 $U G$ 软件 中的 CAM 模块, 图 13、14 给出了球头刀直径分别 为 $10 \mathrm{~mm}$ 和 $14 \mathrm{~mm}$ 时, 按照残留高度为 $0.1 \mathrm{~mm}$ 时 生成的刀位轨迹。从表 1 中可以看出利用本文方法 求解的刀具加工给定曲面, 轨迹长度缩短了 188.2 $\mathrm{mm}$, 加工效率提高了 $12.74 \%$, 同时, 单点处的加 工行宽也增加了 $0.344 \mathrm{~mm}$ 。进一步证明了本文算法 的可行性和高效性。刀具直径增大后可采用更大的 切削参数, 有利于提高工艺过程稳定性。

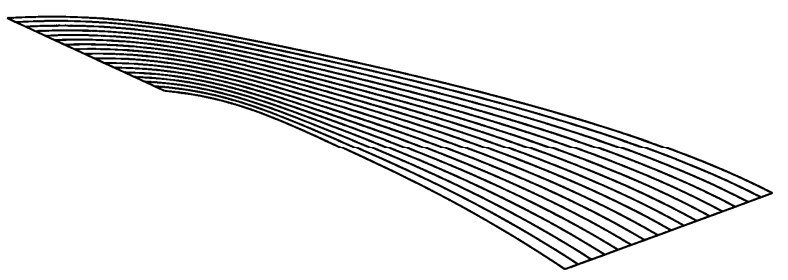

图 13 刀位点轨迹(刀具直径 $10 \mathrm{~mm}$ )

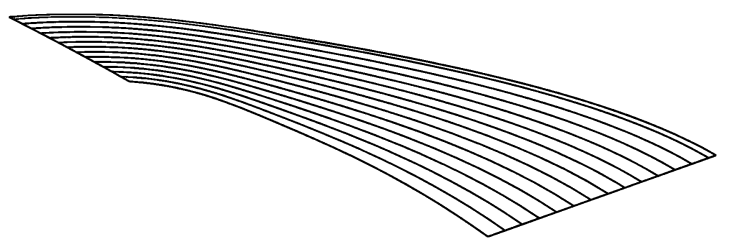

图 14 刀位点轨迹(刀具直径 $14 \mathrm{~mm}$ )

表 1 试验结果比较

\begin{tabular}{ccc}
\hline 刀具直径 $/ \mathrm{mm}$ & 10 & 14 \\
刀触点轨迹数 & 15 & 13 \\
刀触点轨迹总长度 $/ \mathrm{mm}$ & 1477.4 & 1289.2 \\
单点处加工行宽 $/ \mathrm{mm}$ & & \\
$u=0.110, v=0.289$ & 1.923 & 2.267 \\
\hline
\end{tabular}

为进一步验证本文方法的正确性及有效性, 以 整体叶轮通道模型为验证对象, 叶轮高度为 100 $\mathrm{mm}$, 其叶片面为三维空间自由曲面, 叶片面高度 $40 \mathrm{~mm}$, 通道最窄处宽度为 $16.8 \mathrm{~mm}$, 进给速率 250 $\mathrm{mm} / \mathrm{min}$ 。利用 UG 软件中的 CAM 模块生成数控加 工代码, 在 VERICUT 软件中进行仿真验证, 如图
15、16 所示, 刀具切削时间分别为 $311 \mathrm{~s}$ 和 $269 \mathrm{~s}$, 加工时间缩短了 $13.50 \%$ 。应该说明的是, 前面算例 分析中已经指出本文算法加工效率提高 $12.74 \%$, 与 此处有所差别, 这是因为表 1 中的轨迹是在单一叶 片面上规划的, 而此处仿真加工是在叶轮通道内进 行的, 需考虑刀具与轮琼面之间的碰撞干涉, 从而 总轨迹长度缩小, 因此计算数据有所不同。仿真结 果同样验证了本文方法的正确性和高效性。

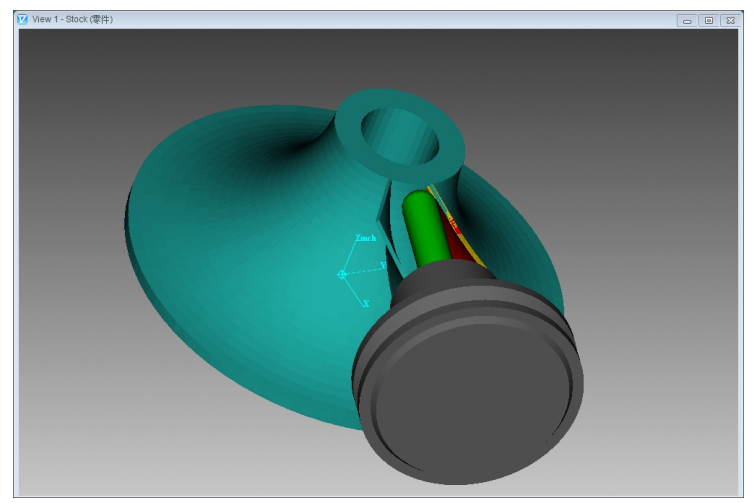

图 15 刀具直径 $10 \mathrm{~mm}$

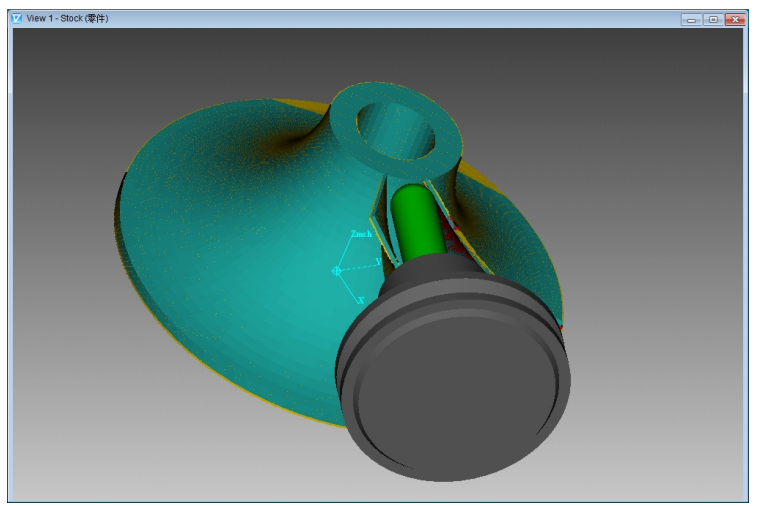

图 16 刀具直径 $14 \mathrm{~mm}$

\section{5 结论}

本文针对球头刀多坐标加工复杂曲面通道零件 无碰撞干涉的合理刀具选择问题, 提出了一种基于 可行空间分析的处理方法。通过分析多轴加工刀具 运动几何约束, 在不考虑刀具尺寸的情况下, 基于 临界约束条件计算了可行摆刀域。通过分析球头刀 可达区域与可行摆刀域之间的关系, 提出一种最大 刀具直径计算方法, 以可行摆刀域为基础, 计算了 最短刀具安全长度。以整体叶轮通道为例, 对本文 方法进行了分析与验证, 验证结果与实际情况相符, 证明了本文方法的正确性和有效性。

\section{参 考 文 献}

[1] LEE Y S, CHANG T C. Automatic cutter selection for 5 -axis sculptured surface machining[J]. International 
Journal of Production Research, 1996, 34(4): 977-998.

[2] GENG L, ZHANG Y F, FUH J Y H. A neural network based approach to 5-axis tool-path length estimation for optimal multi-cutter selection[J]. Computer-Aided Design and Applications, 2011, 8(2): 301-313.

[3] LI LL, ZHANG Y F. Cutter selection for 5-axis milling based on surface decomposition[J]. International Conference on Control, Automation, Robotics, 2004(3): 1863-1868.

[4] DING X, FUH J, LEE K. Interference detection for 3-axis mold machining[J]. Computer-Aided Design, 2001, 33(8: 561-569.

[5] JENSEN CG, RED WE, PI J. Tool selection for five-axis curvature matched machining[J]. Computer-Aided Design, 2002, 34(3): 251-266.

[6] LI LL, ZHANG Y F. Cutter selection for 5-axis milling of sculptured surfaces based on accessibility analysis[J]. International Journal of Production Research, 2006, 44(16): 3303-23.

[7] GLAESER G, WALLNER J. Collision-free 3-axis milling and selection of cutting tools[J]. Computer-Aided Design, 1999, 31: 225-232.

[8] YANG D, HAN Z. Interference detection and optimal tool selection in 3-axis NC machining of free-form surfaces[J]. Computer-Aided Design, 1999, 31: 303-315.

[9] MORIMOTO K, INUI M. A GPU based algorithm for determining the optimal cutting direction in deep mold machining[J]. International symposium on assembly and manufacturing, 2007: 203-208.

[10] LASEMI A, XUE Deyi, GU Peihua. Recent development in CNC machining of freeform surfaces: A state-of-the-art review[J]. Computer-Aided Design, 2010, 42(7): 641-654.

[11] RAO A, SARMA R. On local gouging in five-axis sculptured surface machining using flat-end tools[J]. Computer-Aided Design, 2000, 32(7): 409-420.

[12] WANG N, TANG K. Automatic generation of gouge-free and angular velocity-compliant five-axis tool path $[\mathrm{J}]$. Computer-Aided Design, 2007, 39(10): 841-852.

[13] 尹周平, 丁汉, 熊有伦. 基于可视雉的可接近性分析方 法及其应用[J]. 中国科学，2003.33(11)：979-989.

YIN Zhouping, DING Han, XIONG Youlun. Accessibility analysis algorithm and application based on visibility cone[J]. Science China: Technological Sciences, 2003, 33(11): 979-989.

[14] CHEN Li, XU Ke, TANG Kai. Collision-free tool orientation optimization in five-axis machining of bladed $\operatorname{disk}[\mathrm{J}]$. Journal of Computational Design and Engineering, 2015, 13(4): 197-205.

[15] 张定华. 多轴 NC 编程系统的理论、方法和接口研究 [D]. 西安：西北工业大学，1989.

ZHANG Dinghua. Study on the theory, method and interface for multi-axis NC programming system[D]. Xi'an: Northwestern Polytechnical University: 1989.

[16] 王晶, 张定华, 吴宝海, 等. 基于临界约束的四轴数控 加工刀轴优化方法 $[\mathrm{J}]$. 机械工程学报, 2012，48(17) : 114-120.

WANG Jing, ZHANG Dinghua, WU Baohai, et al. Tool orientation optimization method in four-axis $\mathrm{CNC}$ machining based on critical constraints $[\mathrm{J}]$. Journal of Mechanical Engineering, 2012, 48(17) : 114-120.

[17] 易兵, 刘振宇, 谭建荣. 基于高斯映射的 CAD 网格法 向聚类分割方法 $[\mathrm{J}]$. 机械工程学报，2015，51(7)： 115-123.

YI Bing, LIU Zhenyu, TAN Jianrong. An algorithm for segmenting CAD meshes based on the Gaussian map $[\mathrm{J}]$. Journal of Mechanical Engineering, 2015, 51(7): 115-123.

作者简介：吴宝海(通信作者), 男, 1975 年出生，教授。主要研究方向 为自由曲面多轴加工、智能加工。

E-mail: wubaohai@nwpu.edu.cn

梁满仓, 男, 1994 年出生。主要研究方向为复杂曲面的计算机辅助几何 设计及多坐标数控加工理论。

E-mail: 1mc@mail.nwpu.edu.cn 